\title{
Using cognitive learning theory to design effective on-line statistics tutorials
}

\author{
VICTORIA L. ROMERO, DALE E. BERGER, \\ MICHAEL R. HEALY, and CHRISTOPHER L. ABERSON \\ Claremont Graduate University, Claremont, California
}

\begin{abstract}
Careful attention to principles of learning can improve the design of Web-based lessons and tutorials. Tutorials from the Web Interface for Statistics Education (WISE; http://wise.cgu.edu) demonstrate how specific principles can be integrated into Web design to enhance learning in two areas. First, the impact of students' poor self-regulation abilities on Web-based learning is considered. Second, evidence that specific types of visual presentations improve learning is discussed. Finally, the need for empirical evaluation is emphasized. Specific research and examples from the WISE project are used to illustrate each of these points.
\end{abstract}

Hundreds of sites are available on the WWW to help students learn about statistics. However, not all of these sites are equally helpful. As more people come to rely on Web resources as teaching tools, it is important that we consider what distinguishes effective instructional Web applications. Web Interface for Statistics Education (WISE) is a WWW site (http://wise.cgu.edu) offering support for teaching and learning statistics, featuring interactive statistics tutorials. The design of these tutorials reflects empirically supported learning principles to provide students with the experiences they need to develop a conceptual understanding of statistics. We have previously considered the importance of using constructivist teaching techniques and discovery-based learning (Aberson, Berger, Emerson, \& Romero, 1997; Romero, Berger, \& Aberson, 1997; Romero, Berger, Aberson, \& Healy, 1998). In this paper, we will focus on techniques that support students' self-regulation and awareness of their learning.

The first section of this paper focuses on how WISE uses specific teaching techniques to counteract weaknesses in students' self-regulation abilities. These techniques work by eliciting elaborative thinking, compelling students to assess their own learning, forcing students to confront their misconceptions, and helping students associate new material with familiar concepts. The second section addresses the benefits of the multimedia capabilities of Webbased tutorials: complementary visual and verbal presentations, and physically interactive visual displays. The

The Web Interface for Statistics Education (WISE) is an ongoing project at Claremont Graduate University. It is being developed in collaboration with students and faculty across the Claremont Colleges. WISE is supported by a grant from the Mellon Foundation. The authors thank Sandra Stuckey, Victoria Stewart, and an anonymous reviewer for their insightful reviews of this paper. Correspondence should be addressed to V. L. Romero, Department of Psychology, Claremont Graduate University, 123 E. 8th St., Claremont, CA 91711 (e-mail: victoria. romero@cgu.edu). third section emphasizes the need to evaluate Web-based tutorials and summarizes an empirical evaluation of a WISE tutorial.

\section{Self-Regulation}

The self-paced, independent nature of on-line tutorials offers great flexibility to students. However, this flexibility has the potential to magnify problems associated with a lack of self-regulated learning skills. These skills include selecting effective study strategies, being aware of how well material has been learned, and allotting time and effort appropriately (Hofer, Yu, \& Pintrich, 1998). Although we might expect experienced students to regulate their own learning skillfully, even college students generally are not good self-regulated learners. They often adhere to inefficient learning strategies developed in high school or grade school and fail to adapt their strategies to the college environment (Hofer et al., 1998). In the traditional classroom environment, instructors can pace their lectures to fit their students' progress, but when students are left on their own with an on-line tutorial, they may not have the skills needed to pace themselves appropriately. Without an instructor's intervention, some students will be unlikely to assess their progress accurately and adjust their strategies accordingly.

Elaborative processing. Hofer et al. (1998) noted that one particular weakness in self-regulation abilities is the failure to engage in elaborative thinking. Elaborative processing has long been known to aid in comprehension and retention of conceptual material (Park \& Hanafin, 1993). In statistics, elaborative processing can be encouraged by forcing students to reflect on the concepts they have learned. To promote elaborative processing, WISE concludes each tutorial with several questions that require students to explain the concepts they have learned. For example, one question asks a student to explain a finding that a very large difference between means resulted in a nonsignificant $t$ test. (In this case, it is because the 
variance is also very large.) These reflective exercises encourage students to process the content deeply and integrate the new concepts with their existing knowledge of statistics and research.

Self-monitoring. An inability to monitor one's own progress accurately can be a major obstacle to learning (Bjork, 1995). This is particularly true of Web-based learning; students who mistakenly believe they have mastered the material expend less effort on portions of the tutorial that could have helped them. Bjork reported several aspects of the learning environment that can lead students to overestimate their mastery. One of his most interesting findings is the effect of lesson difficulty. Students feel as though they have learned the most from lessons that are relatively easy and the least from lessons that are difficult. However, the students' perceptions are the reverse of reality; students actually learn more from difficult lessons than from easy lessons. Because students do not feel they are learning from difficult lessons, they are likely to become frustrated and even resentful toward these exercises. For this reason, it is necessary to carefully consider the level of difficulty in the tutorial. If the tutorial is not difficult enough, students may overestimate their mastery of the material. However, if the tutorial is too difficult, students feel as though they are not learning anything. This can lead to feelings of resentment for being forced to "waste time" on lessons that do not help them.

The inability of students to monitor their progress accurately is particularly troublesome in statistics (Lan, 1998). Some students confuse computational proficiency with conceptual mastery. These students mistakenly believe they are doing well because they can use formulas to compute correct answers. However, these same students often lose sight of what these formulas mean. Lan found that even his graduate students were "losing their sense of what they were doing in the class" (p. 87). For these reasons, effective instruction should include devices to force the students to reflect realistically on their progress.

We made several efforts to counter these problems in the WISE tutorials. First, we tried to make the consequences of an incorrect answer as painless as possible for students. We emphasize that there are no penalties for incorrect answers, and no record is kept of how many mistakes are made. Our goal is to limit anxiety and help students to view the tutorial in a favorable light. Our reflective thinking questions are designed to be challenging, but not impossible. We expect most students to be able to answer these questions after using the tutorial. Because these questions are perceived as difficult, students will recognize that they have learned new concepts when they are able to answer these questions.

Confronting students' misconceptions. Bjork (1995) has shown that we are subject to several "illusions of knowing." These illusions trick us into feeling that we understand a concept better than we really do. For example, in a study in which college students had to search for con- tradictions within passages and then rate their comprehension for those passages, Glenberg, Wilkinson, and Epstein (1982) found that up to $51 \%$ of students rated their comprehension as high, even when they had failed to notice obvious contradictions. The students' failure to recognize contradictions that rendered the passage incomprehensible indicates that their comprehension was much lower than they perceived.

Another way to alert students when they have not mastered concepts is to make them aware of their misconceptions. This helps students confront and avoid hindsight biases, described by Bjork (1995). Hindsight bias refers to the fact that when we are simply given an answer to a question, we tend to overestimate the likelihood that we could have arrived at that answer on our own. In a sense, we feel as if we "knew it all along." This bias can lead students to believe that material is easier than it is, that the concepts are simple common sense. Hindsight bias reflects overconfidence in one's knowledge, which dissuades students from seeking deeper understanding of poorly understood concepts. As a result, students often do not allot a lesson enough time or effort. By making students aware of their misconceptions, we can help them confront and correct misunderstandings.

A powerful way to correct students' misconceptions is to confront them with empirical evidence that contradicts their beliefs (Garfield, 1995). Because different students have different misconceptions, a classroom demonstration cannot be equally effective for all students. The interactive capabilities of the Web allow the tutorial to confront students with their specific misconceptions. For example, in our Central Limit Theorem tutorial, students are shown a highly skewed bivariate population and asked to predict how the sampling distribution of the mean $(n=100)$ will look. Students are given this task before they are taught that even very non-normal populations yield normally distributed sampling distributions (given a large enough sample size). These questions purposefully elicit incorrect answers from students in order to direct their attention to why their answers were incorrect. Such an exercise requires students to consider which concepts they misunderstood. This helps avoid the "hindsight" problem because it forces the students to recognize their problem areas prior to arriving at the solution. Upon learning that non-normal population distributions can have normal sampling distributions, students cannot conclude that they "knew it all along" because they just gave an answer that proves otherwise.

Integrating new material with familiar concepts. New material is best learned when it can be integrated easily with familiar material. For this reason, it is beneficial to make efforts to associate new material with familiar material when learning. This is not something students are likely to do on their own. Web tutorials can benefit by explicitly reminding students of familiar concepts that relate to new material. In WISE, we include a "prerequisites" page to let students know what they should know before beginning the tutorial. We give students the 
opportunity to review any of these prerequisite concepts before beginning the tutorial. We also begin our tutorials with a few easy questions that reinforce the prerequisite concepts. These techniques are designed to prime relevant material in the students' minds so that they may interpret the tutorial in the context of what they already know. However, review of old material must be limited. A survey of graduate students who used the WISE tutorials indicated that some considered the initial questions to be too basic and a waste of time. Although these criticisms were from statistically advanced students, they do highlight the need to keep review material brief and to allow proficient students to move through the review more quickly.

\section{Multimedia Lesson Presentation}

One of the primary appeals of the WWW is the ability to present material in multimedia formats. Advances in Java, QuickTime, and other Internet applications are continuously improving the quality of multimedia presentations on the Web. Not only do these applications allow us to develop attractive and efficient lessons, they can help us develop more effective lessons. There is evidence that carefully planned visual presentations and physically interactive graphics can enhance students' learning.

Benefits of complementary verbal and visual presentations. Integrating information that is presented simultaneously in two different media enhances learning and problem solving. Paivio's (1971) dual-coding theory postulates that knowledge can be represented in semantic, visual, or dual (both semantic and visual) formats. When knowledge from the semantic and visual representations are integrated into a dual format, comprehension and memory improves. Mayer and Sims (1994) demonstrated one specific way in which this principle can be used in instruction. They provided students with instruction about the path of air throughout the respiratory system. Some students were presented with a 45-sec animation of the respiratory system and a coordinated narration of the air's route through the system. Other students were presented with the animation and narration separately. When students' factual knowledge about the respiratory system was tested, both experimental groups performed better than a control group who received no instruction. However, when the students were placed in the role of a doctor and asked to solve problems with the respiratory system, the students who received the concurrent multimedia presentations generated more creative solutions than the other groups of students.

We have attempted to capitalize on the advantages of multimedia presentation by making liberal use of interactive graphic displays in the WISE tutorials. For example, the Sampling Distribution applet allows students to observe the growth of the sampling distribution as successive sample means are gathered. We do not present the applets in isolation; they are always presented within a rich verbal explanation of the concepts at work. Although Mayer and Sims (1994) found auditory narration to be effective, we elected to use only on-screen text in WISE. Although we believe sound clips would enhance the learning experience, we are concerned that sound clips would be disruptive when used in campus computer labs. We are also concerned with the extra time needed to download sound clips and plug-ins to play them. However, it may be beneficial to include optional sound clips that could be used to supplement the graphics when appropriate.

Benefits of interactive graphics. Learning is enhanced by motor activity, even when the skill being learned is not a motor task. For example, Cohen (as cited in Baddeley, 1990) found that subjects were more likely to remember objects if they performed a physical action on each object (i.e., bouncing a ball or breaking a match). We capitalize on the benefits of motoric activity by incorporating click-and-drag features into WISE. In our earlier applets (such as the Sampling Distribution applet), students interacted by pushing on-screen buttons with their cursor. In our most recent applets, students are also able to act directly on the distributions. In our Power applet, if students would like to see what would happen if the difference between means was increased, they can place their cursor over the experimental distribution and drag it away from the null distribution. A "power thermometer" immediately shows how these changes in effect size affect power. Sample size can be manipulated by dragging up or down in a "sample size thermometer," and the impact on the shape of the sampling distributions, their overlap, and power is seen immediately. Thus, motoric interaction reinforces the relationships between the concepts represented by the objects on the screen and provides a varied set of cues to enhance learning and memory.

\section{Evaluating Lesson Effectiveness}

Unfortunately, instructors and other experts are not necessarily much better than the students are at evaluating how effective a learning tool actually is. Jolicoeur and Berger $(1986,1988)$ examined judgments of students, educators, and educational software experts regarding the effectiveness of several educational software programs that they used or examined in detail. An empirical test of how well students learned from the programs showed that students, educators, and experts all were poor judges of how effective the programs actually were in practice. This study emphasizes how important it is to evaluate Web-based tutorials empirically to be sure that they are effective in promoting learning.

According to Bjork (1995), when informally evaluating tutorials, instructors are likely to be influenced by students' opinions of their learning experience. Because students' impressions may not accurately reflect what they have learned, dependence on students' opinions can lead instructors astray. For this reason, it is imperative that we conduct systematic empirical evaluations of tutorials. If a tutorial is meant to provide supplementary instruction, the evaluation should compare students' knowledge before and after using the tutorial. Any increase in knowl- 
edge would be encouraging for a supplemental tutorial. However, if a tutorial is intended to replace a more traditional method of learning, we need to ensure that the tutorial is at least as effective as the method it will replace or that it has other additional benefits. For example, if a Web tutorial is meant to replace a text assignment, the evaluation should compare students who use the tutorial with those who complete the standard assignment.

The WISE tutorials are designed to replace some portions of lecture and lab instruction. The goal is not to supplant the instructor but to free class time for discussion and deeper explorations of conceptual topics. For this reason, we chose to compare our Sampling Distribution tutorial with a lecture. We conducted an evaluation with students in two introductory statistics classes who had not yet learned about the sampling distribution in class, one from a local community college and one from a California State University. At each school, we randomly assigned students to either the lecture condition or the tutorial condition. Students in the tutorial condition were placed in a computer lab and started on the tutorial. Students in the lecture condition experienced a good lecture and demonstration that we designed. The lecture included the same material as the tutorial. Students were given pretests and posttests to measure their conceptual understanding of the sampling distribution. We found that both groups demonstrated substantial learning, and there were no significant differences between the tutorial and the lecture groups (Aberson, Berger, Healy, Kyle, \& Romero, in press).

\section{Summary}

We gave careful attention to principles of learning as we designed our WISE tutorials, and we believe the tutorials benefited accordingly. Self-regulation of learning is especially important with self-paced on-line tutorials where students may work in isolation. Special steps can be taken in the design of tutorials to facilitate self-monitoring, such as confronting students with their misconceptions and encouraging elaborative processing and integration of new material with existing knowledge. Internet technology also allows on-line tutorials to integrate information from multiple media and to give students the opportunity to interact physically with representations of concepts. In general, we can do much more to take advantage of these capabilities.

However, we recognize that, even as developers, we cannot necessarily predict how well a tutorial will work in practice. Thus, it is necessary to conduct empirical outcome studies to assess the effectiveness of tutorials and to identify unintended consequences. An outcome assessment of one of our tutorials is encouraging in that it demonstrated that Web-based tutorials for even complex concepts can be an effective method of instruction.

\section{REFERENCES}

Aberson, C. L., Berger, D. E., Emerson, E. P., \& Romero, V. L. (1997). WISE: Web Interface for Statistics Education. Behavior Research Methods, Instruments, \& Computers, 29, 217-221.

aberson, C. L., Berger, D. E., Healy, M. R., Kyle, D. J., \& Romero, V. L. (in press). Evaluation of an interactive tutorial for teaching sampling distributions. Teaching of Psychology.

BADDELEY, A. D. (1990). Human memory: Theory and practice. Boston: Allyn \& Bacon

BJORK, R. A. (1995). Memory and metamemory considerations in the training of human beings. In J. M. A. P. Shimamura (Ed.), Metacognition: Knowing about Knowing (pp. 185-205). Cambridge, MA: MIT Press.

Garfield, J. (1995). How students learn statistics. International Statistics Review, 63, 25-34

Glenberg, A. M., Wilkinson, A. C., \& Epstein, W. (1982). The illusion of knowing: Failure in the self-assessment of comprehension. Memory \& Cognition, 10, 597-602.

Hofer, B. K., Yu, S. L., \& Pintrich, P. R. (1998). Teaching college students to be self-regulated learners. In D. H. Schunk \& B. J. Zimmerman (Eds.), Self-regulated learning: From teaching to self-reflective practice (pp. 57-85). New York: Guilford.

Jolicoevr, K., \& BerGer, D. E. (1986). Do we really know what makes educational software effective? A call for empirical research on effectiveness. Educational Technology, 26, 7-11.

Jolicoeur, K., \& Berger, D. E. (1988). Implementing educational software and evaluating its academic effectiveness: Part II. Educational Technology, 28, 13-19.

LAN, W. Y. (1998). Teaching self-monitoring skills in statistics. In D. H. Schunk \& B. J. Zimmerman (Eds.), Self-regulated learning: From teaching to self-reflective practice (pp. 86-105). New York: Guilford.

MAYER, R., \& SiMS, V. (1994). For whom is a picture worth a thousand words? Extensions of dual-coding theory of multimedia learning. Journal of Educational Technology, 86, 389-401.

PaIvio, A. (1971). Imagery and cognitive processes. New York: Holt, Rinehart \& Winston.

Park, I., \& Hanafin, M. (1993). Empirically based guidelines for the design of interactive multimedia. Educational Technology Research \& Development, 41, 63-85.

Romero, V. L., Berger, D. E., \& Aberson, C. L. (1997, April). Learning theory for web hased tutorials. Paper presented at the annual meeting of the Western Psychological Association, Seattle.

Romero, V. L., Berger, D. E., Aberson, C. L., \& Healy, M. R. (1998, April). Incorporating interactive computer applets into statistics education. Paper presented at the annual meeting of the Western Psychological Association, Albuquerque, NM.

(Manuscript received November 1, 1999; accepted for publication January 26,2000 .) 